\title{
Equidad de la educación en El Salvador
}

\section{Alvaro Carrasco Guzmán}

Fundación Empresarial para el Desarrollo Educativo San Salvador, El Salvador aathos@geocities.com-

fedape@sal.gbrn.net
En términos de equidad de oportunidades educativas, la región muestra grandes problemas y lo mismo se comprueba respecto de El Salvador. El análisis de la inequidad educativa en el país se inicia con una descripción general de las líneas de acción fundamentales propuestas por la reforma educativa. Luego se aborda el estudio de las diferencias en el acceso a la educación, la transmisión intergeneracional de las oportunidades educativas y las disparidades en la calidad de la educación. Se señala que la educación tiene una función instrumental reproductora del orden económico, pero que también tiene una función transformadora orientada a la búsqueda de una sociedad más justa. Se plantea que la contribución de la educación al desarrollo tiene límites y se destaca el efecto de la pobreza en las oportunidades educativas y, por lo tanto, en las posibilidades reales de superación de los individuos. Finalmente, se proponen algunas ideas para maximizar el aporte de la educación al desarrollo de El Salvador. 


\section{I}

\section{Introducción}

Este trabajo pretende aportar a la reflexión sobre el tema de la educación y el desarrollo en El Salvador, teniendo en cuenta la desigualdad de oportunidades que se ofrecen a los sectores sociales con diversos niveles socioeconómicos. Tal reflexión tiene importantes implicaciones ideológicas y prácticas a la hora de educar a los salvadoreños, y puede ser útil para el debate en otros países latinoamericanos que muestran patrones similares. El problema de la inequidad educativa, que se analiza aquí en detalle, coincide en muchos aspectos con la realidad de la mayoría de los países latinoamericanos. El vínculo determinante entre inequidad socioeconómica e inequidad educativa devela los límites actuales del aporte de la educación al crecimiento, el desarrollo y la superación de la pobreza. Por otra par- te, el reconocimiento de este vínculo es una primera luz en el redescubrimiento del papel transformador que puede desempeñar la educación en la sociedad.

En esta investigación se hace uso de datos de El Salvador para examinar, desde la perspectiva de la equidad, aspectos relacionados con la cobertura, la calidad y la transmisión intergeneracional de las oportunidades educativas. Los análisis muestran la magnitud del problema y llevan a reflexionar sobre los límites de la contribución de la educación al desarrollo, dadas las condiciones actuales. En la parte final del artículo se ofrecen además algunas ideas para contribuir a maximizar el aporte de la educación al desarrollo de El Salvador.

\section{II}

\section{El papel de la educación y el problema de la equidad}

En buena medida, desde la perspectiva predominante del desarrollo humano y económico se tiende a ver la educación formal, en sus distintos niveles, como un mecanismo fundamental para la promoción de los nuevos individuos productivos que necesita el modelo económico actual.

Aunque se esté de acuerdo en parte con los fundamentos de la relación entre educación y desarrollo, la postura que aquí se adopta concibe la educación en una dimensión más amplia. No es posible entenderla exclusivamente como el mecanismo que asegura la reproducción de un orden económico que, siendo ampliamente aceptado hoy como el más funcional, dista mucho de lograr una sociedad sin pobreza y sin otras lacras sociales. Tampoco se pretende decir que el

$\square$ Una versión más amplia de este trabajo fue preparada originalmente para ser expuesta en la reunión de 1998 de la Asociación de Estudios Latinoamericanos, realizada en Chicago. El autor agradece a Patricia Craig, Irene Flores, David Bronkema y Alberto Barillas sus comentarios al presente texto. modelo neoliberal sea un mecanismo inviable para el desarrollo social: se trata más bien de tomarlo como un punto de llegada de un largo proceso histórico y político, y como un punto de partida para una sociedad que alcance mayores niveles de justicia social.

En esta lógica, la educación no debe considerarse un mecanismo meramente reproductivo, sino fundamentalmente un poder transformador. La educación en América Latina no puede desligarse de la búsqueda de innovaciones y alternativas que permitan mejorar el funcionamiento actual de las sociedades. La educación no es hija del mercado; la ofrecida por el Estado es una conquista social que busca otorgar posibilidades para que todos/as progresen y para que la sociedad se desarrolle en la dirección que sus miembros determinen y no en una sola dirección incuestionable.

Si se define la educación puramente en su relación con la economía se la instrumentaliza, de modo que el asunto se reduce a cuánta educación y de qué tipo se necesita para el desarrollo económico y la su- 
peración de la pobreza. Esta visión, que es a la vez necesaria y parcial, pasa por alto que el acceso a la educación es un derecho humano en virtud del cual las personas pueden llegar a dimensiones del mundo externo e interno que contribuyen a materializar su potencial personal, y que el nivel educativo de la población es en sí una de las aproximaciones al desarrollo humano de un pueblo.

$\mathrm{Al}$ instrumentalizar la educación se corre el riesgo de despolitizarla y desmoralizarla, en favor de la noble causa del desarrollo económico. Claro que, en esta visión, el instrumento sirve para reproducir el modelo; si se supone que el modelo es justo y que con él se asegura el desarrollo y la superación de la pobreza, la instrumentalización de la educación no ofrece problemas. Pero si se supone que el modelo económico es imperfecto, la asepsia de la educación es peligrosa, pues no se cuestiona cuáles son sus fundamentos ni para qué se educa. De hecho, la educación sí responde a fines políticos. Lo que cabe preguntarse es ¿a cuáles? Se ha hecho mucho hincapié en que la educación debe responder al principio de la productividad, y aunque en este artículo no se niegue la importancia de ese punto de partida, nuestra propuesta es que la educación sirva a los principios de justicia y solidaridad. Como los datos lo demostrarán, la realidad social y educativa de los países latinoamericanos, y en particular de El Salvador, es profundamente inequitativa. Por lo tanto, la educación debe apuntar a promover la transformación de los modos de convivencia social, en pos de unos más armónicos y solidarios.

En América Latina, ${ }^{1}$ el problema de la equidad puede comenzar a analizarse desde el fenómeno de la distribución de la riqueza. A modo de ejemplo, en 1994 la población de la región en situación de pobreza alcanzaba al $39 \%$ y aquélla en extrema pobreza al $17 \%$. En El Salvador la situación es mucho más grave, ya que en 1997 las personas bajo la línea de la pobreza llegaban al $52 \%$ de la población y aquéllas en extrema pobreza al 22\% (El Salvador, Dirección General de Estadísticas y Censos, 1998).

Además del problema de pobreza, la región presenta una desigual distribución de la riqueza entre la población. Aunque durante la primera mitad del decenio de 1990 parece haber habido una moderada tendencia a la reducción de la pobreza, la concentración del ingreso ha sufrido pocos cambios, incluso en los países con mayor crecimiento económico y menores

\footnotetext{
${ }^{1}$ La información incluida en esta sección se basa en CEPAL, 1998.
}

niveles de pobreza (CEPAL, 1997a, p. 21). Lo mismo es válido para El Salvador, donde entre 1992 y 1995 la distribución del ingreso se ha mantenido constante, según lo demuestra el índice de Gini de 0.5 para estos años (BID, 1998). Estos índices hacen de El Salvador uno de los países latinoamericanos con mayores diferencias distributivas (véase datos comparados en CEPAL, 1997b, p. 46). Otros indicadores señalan asimismo que la distribución del desarrollo humano en El Salvador se ha dado desigualmente, siendo el progreso de los sectores más pobres menor que el de los sectores más ricos (pNUD, 1997, p. 23).

Centrando el análisis en los aspectos educativos, se observa que los esfuerzos por ampliar la cobertura de la educación no se han traducido en los últimos 10 a 15 años en una disminución de las distancias entre los distintos estratos sociales. Según datos de los años ochenta y mediados de los noventa sobre zonas urbanas de 11 países latinoamericanos, las diferencias en la proporción de jóvenes de 20 a 24 años de edad con 12 años de estudios cursados, provenientes de hogares con distinto capital educativo, se mantuvieron prácticamente invariables.

Otros datos indican que en los países de la región entre el 30 y el $45 \%$ de los hogares pueden ser calificados como vulnerables a la pobreza, mientras que sólo un $10 \%$ de los hogares concentran capital económico y educativo. El $80 \%$ de jóvenes entre 20 y 24 años provenientes de hogares vulnerables a la pobreza se encuentran en situación de rezago o de abandono escolar, situación que sólo se observa en menos del $40 \%$ de jóvenes pertenecientes a hogares que concentran capital económico y educativo.

Además de las inequidades en el acceso a la educación y en los años cursados, las diferencias educativas entre estudiantes de distintos niveles socioeconómicos se agudizan aún más si se toma en cuenta la desigual calidad de la educación que reciben. Un indicador cuantitativo que se usa para aproximarse al fenómeno de la calidad es el rendimiento en pruebas de aprendizaje estandarizadas. Los datos a nivel regional muestran importantes disparidades entre el sector público y el privado; mientras el promedio de los estudiantes del sector público apenas logra el $50 \%$ de lo esperado en el currículo oficial, el promedio de los matriculados en colegios privados logra cerca del 100\% (CEPAL, 1998, p. 70). En Chile, uno de los países con mayor crecimiento económico en los años noventa y con uno de los promedios más altos de América Latina en años de estudios de la población, las pruebas de aprendizaje han mostrado que el factor que más influ- 
ye en el rendimiento es el nivel socioeconómico de la familia de los estudiantes (PIIE/UNICEF, 1995).

Por otra parte, hay una larga tradición que vincula la movilidad social con la oportunidad de acceder a más y mejor educación. Esta relación, indiscutible en la teoría, encuentra importantes escollos en la realidad, siendo uno de los principales la misma condición de pobreza que se desea superar.

En América Latina se ha elevado el promedio de escolaridad de una generación a otra. Sin embargo, es escasa la proporción de jóvenes que se empina significativamente por sobre el nivel educativo de sus padres. Así, mientras en promedio los jóvenes tienen tres años de estudio más que sus progenitores, sólo un tercio de los jóvenes urbanos y un décimo de los rurales han logrado superar el nivel de educación de sus padres y alcanzar a la vez un capital educativo básico de 12 años de estudio (CEPAL, 1998, p. 60). El 47\% de los jóvenes urbanos y el $73 \%$ de los rurales no superan el nivel educativo de sus padres y tampoco alcanzan el umbral educativo básico. ${ }^{2}$

Las cifras que reflejan la transición intergeneracional de las oportunidades encuentran eco también en la opinión de quienes han sido consultados sobre este tema. Algunos datos para Chile, por ejemplo, parecen indicar que la visión de los padres entrevistados con respecto al futuro de sus hijos está en buena medida influida por la calidad del colegio, que a su vez está determinada en gran parte por la posibilidad de acceder a una educación privada pagada (PNUD, 1998, p. 179).

En resumen: "la persistencia de la falta de equidad en el acceso a la educación asociada al estrato social de origen indica que, en gran medida, las oportunidades de bienestar de los actuales jóvenes ya quedaron plasmadas por el patrón de desigualdades prevalecientes en la generación anterior" (CEPAL, 1998, p. 65).

\section{III}

\section{La reforma educativa salvadoreña y las políticas de equidad}

Planteado el contexto latinoamericano, en adelante el análisis se centrará en el caso de El Salvador, que presenta, como se verá, tendencias similares a las de la región. En este país ha existido una endémica situación de inequidad social, que fue una de las causas más importantes de la guerra civil de los años ochenta. Los acuerdos de paz se lograron cuando se enfrentó la necesidad de terminar con la violencia y transformar la sociedad salvadoreña mediante la creación de instituciones democráticas y condiciones de vida más justas para la mayoría de la población. En el plano educativo, la experiencia de reforma de este país centroamericano es particularmente interesante, pues ha contado con un importante apoyo internacional y ha respondido al modelo de desarrollo y de educación predominante en los círculos internacionales.

En 1993, la Universidad de Harvard, la Universidad Centroamericana José Simeón Cañas (UCA) y la Fundación Empresarial para el Desarrollo Educativo (FEPADE) realizaron un diagnóstico del sector de la educación que inició un proceso de diálogo y reflexión en el país sobre la necesidad de reformar el sistema educativo. La investigación mostró las profundas de- ficiencias de este último en términos de acceso y de calidad en todos los niveles, así como la insuficiencia de los recursos públicos destinados al sector educativo y la mala administración que se hacía de los disponibles. Hizo presente también la necesidad de revisar y replantear tanto la legislación educativa como la formación docente. En suma, el informe respectivo señaló la urgencia de emprender cambios radicales en la mayoría de los ámbitos del sistema educativo formal en sus distintos niveles.

Con los aportes de la investigación UCA/Harvard/ FEPADE, el Ministerio de Educación impulsó un proceso de consulta con diversos sectores de la sociedad. Se creó la Comisión de Educación, Ciencia y Desarrollo, que tenía como objetivo presentar lineamientos para

\footnotetext{
${ }^{2}$ Según cálculos de la CEPAL (1997b, pp. 66), el análisis del ingreso laboral de quienes se incorporan al mercado de trabajo en la región indica que, actualmente, completar el ciclo secundario y cursar como mínimo 12 años de estudio es básico para tener acceso al bienestar. En un gran número de países los datos muestran que alcanzar ese umbral educativo se traduce, con una probabilidad superior al $80 \%$, en la percepción de un ingreso que permite salir de la pobreza.
} 
enmarcar un proceso de transformación de la enseñanza en el país. También se hizo una amplia consulta a organizaciones mediante el Foro Consultivo Nacional sobre la Reforma Educativa. La Consulta '95, orientada a recoger la opinión de docentes, de padres y alumnos y de técnicos del Ministerio de Educación, así como la Consulta de ANDES 21 de Junio, la asociación gremial de docentes más importante del país, fueron otros de los procesos de consulta que sirvieron de base para preparar la Reforma.

Con los importantes antecedentes antes mencionados, el Ministerio de Educación planteó su plan de reforma en lo que llamó el Plan Decenal 1995-2005. Este documento postula como objetivos mejorar la calidad de la educación en los distintos niveles; elevar la eficiencia, eficacia y equidad del sistema educativo; democratizar la educación; crear nuevas modalidades de provisión de servicios, y fortalecer la formación de valores humanos y cívicos. Las metas establecidas para el decenio fueron: disminuir las tasas de analfabetismo, de ausentismo docente y de deserción y repitencia escolar; aumentar la cobertura de los servicios educativos; involucrar a la comunidad educativa en la administración de la educación; usar eficientemente los recursos del Estado, y dar solución oportuna a los problemas del establecimiento escolar.

Entre las medidas con que la Reforma Educativa trata de atacar específicamente el problema de la inequidad están: el establecimiento del Programa de Escuela Saludable, y del programa Educación con Participación de la Comunidad (EDUCO), el uso del Programa Mundial de Alimentos, las Aulas Alternativas, y la ampliación de la infraestructura escolar y de la educación a distancia.

En El Salvador, las medidas para ampliar la cobertura han dado prioridad a las zonas rurales y a las más pobres. Como resultado, la cobertura ha aumentado en todos los niveles del sistema de educación no superior, pero en mayor medida en la enseñanza parvularia y básica.

Entre las iniciativas para mejorar la calidad de la educación resaltan el Programa de Escuela Saludable y el programa EDUCO, por responder en su origen a un principio de discriminación positiva. El resto de las medidas en el ámbito de la calidad han sido comunes a todas las escuelas.

\section{IV}

\section{La equidad de la educación en El Salvador}

Pese a las acciones emprendidas por el Ministerio de Educación, en el contexto de la Reforma Educativa, para disminuir las diferencias educativas en la sociedad, las inequidades que se observan entre los distintos sectores sociales son todavía enormes. Reconocer la dramática insuficiencia de los esfuerzos (pese a los importantes logros de los últimos años y el adecuado rumbo tomado), así como las coincidencias de la situación de El Salvador, en términos de equidad, con la de la mayoría de los países latinoamericanos, constituye un primer paso en la reflexión acerca de las medidas iniciadas y la necesidad de buscar nuevas estrategias para superar la situación actual.

\section{Equidad en el acceso}

La situación educativa de la población, medida por los años estudiados, es bastante negativa. Según datos de 1996 (cuadro 1), el 58\% de la población de seis años o más tiene cinco o menos años de estudio, mientras que solamente el $6.1 \%$ ha cursado más de 12 años. Además, la escolaridad se distribuye desigualmente, dependiendo del nivel de ingreso de las personas.

De las personas con cinco años de estudio o menos, un $14 \%$ se encuentra en el cuartil de ingresos más altos y un $32.9 \%$ - el doble de personas- en el cuartil de ingresos más bajos (cuadro 1). Si el análisis se hace por deciles, se encuentra que solamente un $4 \%$ de la población correspondiente al decil más rico tiene cinco años o menos de estudio, mientras que el $14 \%$ de la correspondiente al decil de menos ingresos se halla en tal situación. Por otra parte, si se observan los datos para las personas con más de doce años de estudio, las diferencias son aún mayores; sólo alcanza este nivel educativo el $2.7 \%$ de las personas del cuartil más pobre, mientras que un $77.5 \%$ de las personas del cuartil de mayores ingresos puede acceder a estudios más allá de los secundarios.

Datos de la población en edad preescolar y escolar (4 a 18 años) muestran, para el mismo año, que un 
CUADRO 1

El Salvador: Distribución de la población de 6 años o más, según años de estudio, 1996

(Porcentajes)

\begin{tabular}{|c|c|c|c|c|c|}
\hline \multirow[b]{2}{*}{ Años de estudio } & \multicolumn{5}{|c|}{ (Cuartiles según nivel de ingreso) } \\
\hline & Total & $1^{\mathrm{er}}$ cuartil & $2^{\circ}$ cuartil & $3^{\text {er. }}$ cuartil & $4^{\circ}$ cuartil \\
\hline 0 a 5 años & 58.0 & 32.9 & 28.6 & 24.2 & 14.2 \\
\hline 6 a 9 años & 24.1 & 14.9 & 23.1 & 31.7 & 30.3 \\
\hline 10 a 12 años & 11.7 & 5.3 & 12.8 & 29.7 & 52.3 \\
\hline Más de 12 años & 6.1 & 2.7 & 4.7 & 15.0 & 77.5 \\
\hline Otros $^{\mathrm{a}}$ & 0.0 & 47.4 & 52.6 & - & - \\
\hline
\end{tabular}

Fuente: El Salvador, Dirección General de Estadísticas y Censos (1997).

a Incluye las categorías "educación especial" e "ignorado".

CUADRO 2

El Salvador: Proporción de niños/as que asisten a la escuela, por edades y grupo de ingreso, 1991 y 1996

(Porcentajes)

\begin{tabular}{|c|c|c|c|c|c|c|c|c|c|c|c|c|}
\hline \multirow[t]{2}{*}{ Edad } & \multicolumn{2}{|c|}{5 años } & \multicolumn{2}{|c|}{6 años } & \multicolumn{2}{|c|}{7 años } & \multicolumn{2}{|c|}{13 años } & \multicolumn{2}{|c|}{14 años } & \multicolumn{2}{|c|}{15 años } \\
\hline & 1991 & 1996 & 1991 & 1996 & 1991 & 1996 & 1991 & 1996 & 1991 & 1996 & 1991 & 1996 \\
\hline $1^{\text {er. }}$ quintil & 16 & 33 & 30 & 47 & 55 & 70 & 64 & 74 & 53 & 65 & 45 & 53 \\
\hline $2^{\circ}$ quintil & 20 & 37 & 38 & 54 & 62 & 79 & 70 & 82 & 59 & 67 & 52 & 56 \\
\hline $3^{\text {er. }}$ quintil & 36 & 48 & 54 & 65 & 71 & 80 & 74 & 78 & 72 & 74 & 58 & 68 \\
\hline $4^{\circ}$ quintil & 53 & 64 & 74 & 83 & 84 & 88 & 86 & 85 & 74 & 82 & 70 & 72 \\
\hline $9^{\circ}$ decil & 69 & 83 & 83 & 93 & 90 & 95 & 89 & 90 & 79 & 85 & 81 & 84 \\
\hline $10^{\circ}$ decil & 74 & 93 & 85 & 93 & 91 & 100 & 90 & 98 & 87 & 98 & 82 & 94 \\
\hline
\end{tabular}

Fuentes: Reimers, coord. (1995), p. 71 y El Salvador, Dirección General de Estadísticas y Censos (1997).

$69 \%$ de este grupo se encuentra estudiando, observándose también una desigual distribución según el ingreso. En el quintil más pobre, el $59 \%$ recibe algún tipo de educación, mientras que en el decil más rico la cifra es de 89\% (El Salvador, Dirección General de Estadísticas y Censos, 1997).

En un análisis más detallado de las desigualdades en el acceso a la educación según el ingreso de los hogares (cuadro 2) se consideran algunos grupos de edad y se compara su evolución en cinco años (entre 1991 y 1996). Los datos para 1996 muestran que nueve de cada 10 niños/as de cinco años de edad provenientes de los hogares con mayores ingresos se encuentran estudiando, muy probablemente en la educación preescolar, mientras que solamente pueden estudiar tres de cada 10 niños/as del quintil de menores ingresos. Entre los/las adolescentes de 15 años, se mantiene prácticamente la misma proporción para aquellos/as pertenecientes al decil de mayores ingresos y se produce un ligero incremento para el $20 \%$ de la población de menores ingresos, pues estudian cinco de cada diez niños/as.
Es posible observar además que en casi todas la edades consideradas y en los distintos niveles de ingreso ha aumentado el acceso a la educación. El incremento ha sido mayor para las edades de cinco, seis y siete años, así como para los dos primeros quintiles de ingreso y el décimo decil, y ha favorecido en mayor medida a los niños/as más pobres, sobre todo a aquellos en edad preescolar y que se inician en el sistema escolar oficial. También ha sido importante para los niños provenientes del decil con mayores ingresos, pero en estos casos el aumento ha sido más pronunciado para los de cinco, 14 y 15 años. Ha habido destacables avances en el acceso a la educación básica desde 1991: en los sectores más ricos han sido particularmente significativos en las edades correspondientes a los últimos grados de la enseñanza básica, y en los sectores más pobres han sido mayores en las edades de educación preescolar y primeros grados de educación escolar.

Entre 1991 y 1996, la diferencia en el acceso al estudio entre el primer quintil de ingreso y el décimo decil para la edad de siete años ha decrecido, pasando 
del 36 al 30\%; esto indica que el avance ha sido mayor en los grupos más pobres, disminuyendo así las desigualdades. Sin embargo, la diferencia aumenta progresivamente al subir las edades, llegando a ser de 41\% para el grupo de 15 años. Esta situación se explica en parte porque las medidas para ampliar la cobertura emprendidas por el Ministerio de Educación han estado orientadas mayoritariamente al primer ciclo de enseñanza básica ( $1^{\circ}$ a $6^{\circ}$ grados). El análisis de la evolución de la matrícula reportada por ese ministerio también pone de relieve significativos avances de cobertura en el nivel básico, y menores logros en la educación secundaria (Carrasco y Fernández, por publicarse).

Otro ámbito de análisis en el cual es posible constatar inequidades de acceso a la educación está dado por la naturaleza urbana o rural de la zona donde se habita. El acceso al estudio para el total de la población entre 7 y 18 años de edad es de $74 \%$, pero el análisis por zonas muestra que en todas las edades un $83 \%$ de los niños/as y jóvenes de hogares urbanos estudia, mientras que en el área rural lo hace sólo el 66\%. Además, igual que en la comparación por sectores de ingreso, las diferencias observadas entre las zonas urbanas y rurales son mayores en los últimos años del tramo de edad de siete a 18 años. El sistema se hace más inequitativo a medida que se avanza en las edades y los grados educativos (FEPADE, 1997).

En los niveles superiores del sistema educativo, las disparidades se mantienen. Así, en 1996, de 100 jóvenes que asisten al $2^{\circ}$ año de enseñanza media, 40 provienen del decil de mayores ingresos y 10 del de menores ingresos. Entre quienes informan estar realizando el primer año de estudios universitarios, solamente el $2.5 \%$ corresponde al cuartil de menores ingresos y el $62 \%$ se concentra en el cuartil más rico. De todos los estudiantes universitarios, solamente el $2 \%$ proviene del quintil de más bajos ingresos, y el $30 \%$ del decil de mayores ingresos; no se observan variaciones significativas entre dichas cifras y las de 1991, que fueron de $2.35 \%$ y $31.67 \%$, respectivamente (Reimers, coord., 1995, p. 72).

En cuanto a las diferencias por géneros, se verifica una situación educativa desfavorable para la mujer en la población general. Al considerar en el análisis a la población general, se incluyen segmentos que son producto de sistemas educativos anteriores que discriminaban en alto grado a las mujeres. Sin embargo, la situación de las niñas y jóvenes en el sistema educativo actual muestra un panorama equitativo entre varones y mujeres. Según datos hasta 1995, mientras en el grupo poblacional de 60 años o más, que no había aprobado ningún grado, existía un 36\% de diferencia en detrimento del sexo femenino, en el grupo
GRAFICO 1

El Salvador: Vinculación a la oferta/demanda de educación de las razones para no estudiar, por grupos de edades

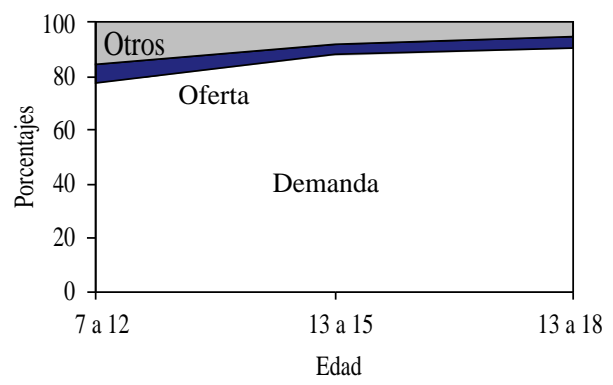

Fuente: FEPADE (1998, p. 76).

poblacional de 6 a 9 años la diferencia era solamente de un $1 \%$, pero ahora en favor del sexo femenino $(\mathrm{Ca}-$ rrasco y Fernández, por publicarse). Esta igualdad entre géneros se comprueba también en las cifras de analfabetismo, así como en el porcentaje de personas de distintas edades que se encuentran estudiando (FEPADE, 1997).

En la búsqueda de razones que expliquen las diferencias de acceso a la educación entre los distintos sectores poblacionales según el ingreso y el tipo de zona de residencia, la respuesta intuitivamente hace referencia a la pobreza de los que no estudian. Esto es respaldado por los datos (El Salvador, Dirección General de Estadísticas y Censos, 1997), ya que las cuatro causas para no estudiar que se mencionan con mayor frecuencia son, en orden decreciente: "muy caro" (falta de recursos económicos), "trabajo", "causas del hogar" y "no vale la pena". Las cuatro razones están vinculadas a las carencias de la población. Las respuestas indican que las razones para no estudiar van fundamentalmente por el lado de la poca demanda más que por el de la naturaleza y estructura de la oferta. Además, los argumentos relacionados con la oferta son más frecuentes en los primeros grupos de edades, y a medida que las edades avanzan se concentran aún más en torno a la poca demanda (FEPADE, 1998, p. 76).

Según una encuesta de opinión aplicada por la UCA en 1997, el 19\% de los menores de 19 años no estaba estudiando. El 46\% de los entrevistados justificó esta situación diciendo que no tenían dinero/recursos; el $28 \%$ dijo que los menores no estudiaban porque tenían que trabajar (incluyéndose labores domésticas); el $18 \%$ hizo referencia a que no tenían deseos de estudiar $\mathrm{y}$ un $7 \%$ dio otras respuestas. Estos datos muestran que para el $73 \%$ de los entrevistados, la principal causa que limita el acceso a las escuelas es la pobreza. 


\section{La transmisión intergeneracional de las opor- tunidades educativas en El Salvador ${ }^{3}$}

Según datos de 1997, al comparar el promedio de años de estudio de los jóvenes entre 20 y 24 años de edad con el de sus padres, se comprueba un incremento de 4.7 años; el promedio para los padres es de 4.2 años, mientras que para los hijos es de 8.9 años. En las zonas urbanas este incremento ha sido de cinco años, al pasar de seis años de estudio para los padres a 11 para los hijos; en las zonas rurales también ha habido un incremento, aunque menor, desde un promedio de dos años de estudio para los padres a 5.8 para los hijos entre 20 y 24 años. Esto significa que aunque ambos sectores han progresado, el aumento de los años de estudio ha sido mayor en las zonas urbanas, por lo que se han acentuado aún más las diferencias con las zonas rurales.

Ahora bien, el incremento medio en los años de estudio se da por la contribución que hace el $49 \%$ de jóvenes que logra superar el nivel educativo de sus padres, ya que el otro 51\% no lo ha superado. Dentro de estas dos categorías, es importante identificar además qué porcentaje de la población salvadoreña ha logrado sobrepasar el umbral educativo básico que aumenta la probabilidad de generar ingresos suficientes para vivir dignamente. En esta línea de análisis se encontró que sólo el $4.4 \%$ de la población entre 20 y 24 años no supera la educación de sus padres pero logra estudiar 12 años o más. Mientras tanto, un $46 \%$ de los jóvenes no supera la educación que sus padres recibieron ni alcanza el umbral educativo básico para acceder al bienestar. Solamente el 36.6\% de los jóvenes logra superar el nivel educativo de sus padres y a la vez adquirir un capital educativo básico: sólo ellos tendrán posibilidades de movilidad social ascendente basada en el capital educativo. Además, un $12.7 \%$ de los jóvenes supera el nivel educativo de sus padres, pero no lo suficiente para tener siquiera 12 años de estudio.

También la situación es inequitativa entre zonas urbanas y zonas rurales. Un $51 \%$ de los jóvenes urbanos logra superar el nivel educativo de sus padres y simultáneamente cursar 12 años de estudio, mientras que sólo el $16 \%$ de los jóvenes rurales logra hacerlo (gráfico 2). Además, el 73\% de los jóvenes de zonas rurales no supera el nivel educativo de sus padres ni tampoco alcanza a estudiar 12 años, y un 11\% adicio-

\footnotetext{
${ }^{3}$ El procedimiento para calcular la superación educativa se hizo replicando el modelo desarrollado por la CEPAL (1998, p. 61).
}

GRÁFICO 2

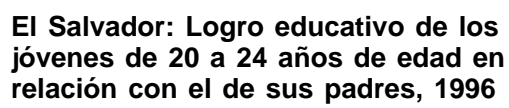

A. Zona urbana

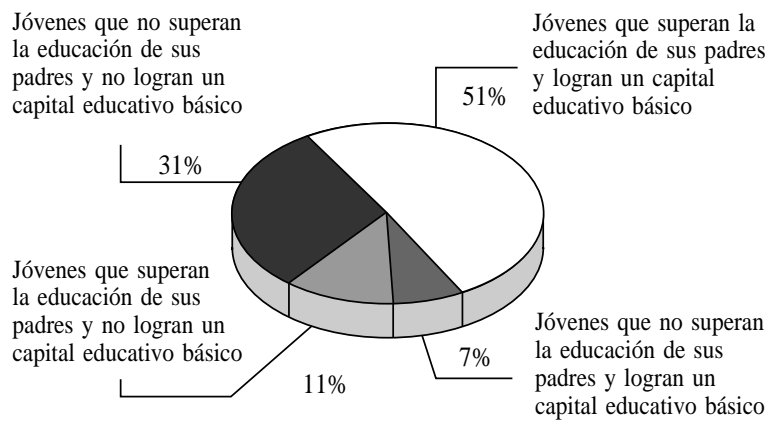

B. Zona rural

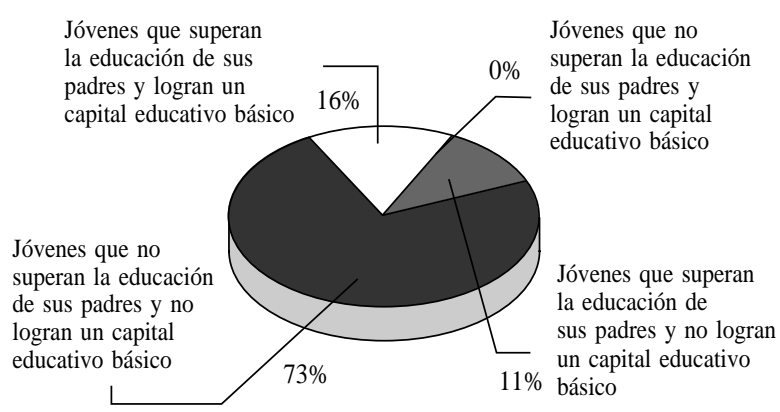

Fuente: El Salvador, Dirección General de Estadísticas y Censos (1997).

nal, si bien supera la educación de sus padres, no logra adquirir un capital educativo básico.

Los datos entregados indican que en El Salvador existe una baja superación educativa intergeneracional, sobre todo en los sectores pobres de la sociedad. Para buena parte de la población, existen pocas posibilidades de superar los niveles educativos de los padres y lograr un capital educativo que le permita acceder a mejores condiciones de vida. Los logros educativos de las nuevas generaciones en términos de años de estudio están determinados en buena medida por la estructura de desigualdades de la generación anterior.

\section{Equidad en la calidad}

Para un gran número de personas, el mejoramiento de sus condiciones de vida se ve limitado por sus escasas posibilidades de acceder a la escuela. Sin embargo, el sistema educativo, a pesar de persistentes problemas de equidad, tiende a ir elevando su cobertura. Aunque 
todavía la tarea de posibilitar el acceso a la educación es un reto enorme para la sociedad, el tema de la calidad es también de particular interés para quienes, habiendo accedido al sistema escolar, aspiran a recibir una educación lo suficientemente buena como para permitirles mejorar las condiciones de vida de sus familias de origen. En El Salvador, las diferencias en la calidad de la educación son enormes, como muestran a continuación los resultados de las pruebas de rendimiento y la repitencia.

\section{a) Diferencias de rendimiento en las Pruebas de Logros de Aprendizaje}

Una de las acciones que acompañó a la elaboración del nuevo currículo fue la elaboración de las Pruebas de Logros de Aprendizaje (PLA) y su aplicación desde 1993 a una muestra de alumnos/as de $3^{\circ}$ y $5^{\circ}$ grados. La prueba evalúa y refleja en un puntaje el cumplimiento de los objetivos de los programas oficiales. En matemáticas el puntaje máximo posible era de 10 $\mathrm{y}$ en lenguaje de 9.

Según datos de 1994 y 1995, el promedio de los alumnos/as de $3^{\circ}$ y $5^{\circ}$ grado de las escuelas del país no dominaba, en el mejor de los casos, ni siquiera el $40 \%$ de los objetivos de aprendizaje en las materias de lenguaje y matemáticas. Análisis complementarios de carácter comparativo mostraban que los alumnos/as de las zonas urbanas habían alcanzado un promedio levemente mayor que los de zonas rurales, y que el rendimiento de los alumnos/as en escuelas EDUCO era aún menor que el de aquellos/as en escuelas rurales no EDUCo (El Salvador, Ministerio de Educación, 1996).

Ahora bien, el rendimiento de los alumnos/as, al igual que el acceso a la escuela, varía según el nivel de ingreso de las familias. A modo de ejemplo, las comparaciones entre las escuelas públicas y privadas también reflejan la tendencia observada en otros países. En matemáticas, la media de los puntajes de los alumnos/ as de escuelas privadas es 2.15 puntos más alta que la media de los puntajes de alumnos/as de escuelas públicas, y en lenguaje, la ventaja de las escuelas privadas es de 1.5 puntos. $^{4}$

Finalmente, un estudio realizado por profesionales del Ministerio de Educación y de la FEPADE (FEPADE/

\footnotetext{
${ }^{4}$ La diferencia de medias es estadísticamente significativa para ambas materias. El sPSs (Statistical Package for Social Sciences) arrojó que $\mathrm{P}=0.000$. Estos análisis fueron hechos con información contenida en la base de datos de las Pruebas de Logros de Aprendizaje aplicadas en 1996.
}

El Salvador, Ministerio de Educación, 1998) examinó, mediante un análisis de regresión múltiple, los factores que influyen en los rendimientos en las PLA. En el modelo se incluyó un conjunto de factores alterables por las acciones del sistema educativo y un grupo de factores no alterables directamente por las acciones inmediatas del sistema educativo. Entre las variables se hallaban la experiencia docente, el nivel educativo del docente, un indicador económico de la familia y un indicador de desarrollo por municipio (indicador de necesidades básicas insatisfechas por municipio). El modelo en lenguaje tuvo un $\mathrm{R}^{2}=0.28$ y en matemáticas un $\mathrm{R}^{2}=0.30 .{ }^{5}$ Según este análisis de regresión, los alumnos de escuelas privadas tienden a obtener 0.33 puntos más que los del sector público en lenguaje y 4.0 puntos más en matemáticas. Además, a partir de los resultados del análisis de regresión es posible calcular que en matemáticas los alumnos provenientes de uno de los municipios más pobres (con más necesidades básicas insatisfechas) logran 3.02 puntos menos que los alumnos de uno de los municipios más ricos.

El estudio citado concluye que "en ambas materias los indicadores que individualmente contribuyen en mayor medida a explicar el rendimiento en las Pruebas de Logros de Aprendizaje, fueron aquellos relacionados con las condiciones sociales, económicas y culturales de las poblaciones que asistían a estas escuelas (sector y zona de la escuela, condiciones socioeconómicas de las familias, niveles de pobreza de los municipios donde están ubicadas las escuelas). Los alumnos/ as de familias con niveles socioeconómicos inferiores pertenecientes a escuelas del sector público y de municipios más pobres se encuentran en desventaja en términos del rendimiento" (FEPADE/El Salvador, Ministerio de Educación 1998, p. 2).

Estos datos indican que, en general, el rendimiento de los alumnos salvadoreños, medido por las actuales pruebas de aprendizaje, es muy bajo. Además, demuestran que, al igual que en la mayoría de los países latinoamericanos, el rendimiento de los alumnos más pobres es mucho más bajo que el de aquellos/as con una mejor situación socioeconómica. Los alumnos/as provenientes de los estratos socioeconómicos más altos aprenden más y de esta manera se encuentran mejor preparados para aprovechar las oportunidades educativas secundarias y terciarias y para desempeñarse laboralmente en el futuro.

\footnotetext{
${ }^{5}$ Ambos modelos son estadísticamente significativos: $\mathrm{F}=0.000$.
} 
Las opiniones de la población respaldan los análisis anteriores, ya que mientras el $54 \%$ de las personas de nivel socioeconómico alto expresa encontrarse muy satisfecho con la educación que reciben los menores residentes en su hogar, entre los entrevistados del sector marginal solamente el $30 \%$ expresa el mismo grado de satisfacción. Además, el $44 \%$ de los entrevistados cree que la educación privada es mejor que la pública, mientras que la opinión contraria es sostenida solamente por el 28\% (IUDOP/UCA, 1997).

\section{b) Repitencia}

Otro indicador comúnmente utilizado para aquilatar la calidad de la educación son las tasas de repitencia. Según la Encuesta de Hogares de Propósitos Múltiples, el 8.8\% de los estudiantes en edad escolar estaba repitiendo grado en 1996. Pero en esta repitencia también se observaba una distribución desigual (cuadro 3). Así, para los estudiantes pertenecientes al primer quintil de ingreso la tasa de repitencia era de $13.2 \%$, y el porcentaje de repitentes iba disminuyendo progresivamente de un quintil de ingreso a otro, hasta que en el decil de mayores ingresos solamente el $3.9 \%$ de los estudiantes eran repitentes.

$\mathrm{El}$ análisis por área de residencia también refleja inequidad, ya que en la zona rural repite grado el $12 \%$ de los estudiantes, mientras en la zona urbana sólo lo hace el $6.3 \%$. Igualmente, entre los alumnos de colegios privados la tasa de repitencia alcanzó al $5.1 \%$ y en las escuelas públicas al $9.8 \%$.

Al comparar la repitencia por género, se encuentra que los niños repiten más que las niñas: $9.7 \%$ contra $7.9 \%$. Como en el acceso a la escuela, tampoco en la repitencia se observa una situación desfavorable para las niñas.

Las datos demuestran que son los niños/as más pobres los que tienen más problemas para cumplir con

CUADRO 3

El Salvador: Estudiantes repitentes, por grupos de ingreso, 1996

(Porcentajes)

\begin{tabular}{lc}
\hline Grupo de ingreso & $\%$ que repite \\
\hline Primer quintil & 13.2 \\
Segundo quintil & 11.1 \\
Tercer quintil & 8.1 \\
Cuarto quintil & 6.5 \\
Noveno decil & 4.4 \\
Décimo decil & 3.9 \\
\hline
\end{tabular}

Fuente: El Salvador, Dirección General de Estadísticas y Censos (1997). los requisitos de aprendizaje mínimos que la escuela exige para promover a sus alumnos. En general, los niveles de exigencia de las escuelas públicas son, en términos absolutos, menores que los de las escuelas privadas y, a pesar de ello, hay más repitentes. Estos parecen indicar que los padres más pobres y las escuelas públicas tienen menos variantes para apoyar las dificultades de aprendizaje de los niños/as, por lo cual éstos exhiben mayores niveles de repitencia, demoran más en completar la enseñanza básica y tienen más probabilidades de desertar; así, es mayor el desperdicio de recursos de la familia y del Estado en los niños que repiten. En cambio, los niños/as de familias con mayores ingresos tienen más facilidad para completar sus estudios, lo hacen en menos tiempo y desperdician menos recursos.

\section{c) Las prácticas educativas}

En una investigación realizada por el Programa de Formación de Investigadores Educativos en 1997 (PROFINE, 1997) se llevaron a cabo observaciones de las prácticas pedagógicas de aula en 140 escuelas públicas. En ellas se vio que la mayoría de las prácticas educativas que todavía predominan en las aulas ubican al profesor en el centro de la enseñanza. Además, aunque se observaron algunas prácticas de juegos y trabajo en grupo que son incipientes esfuerzos por responder a las recomendaciones de la Reforma Educativa, faltan dominio teórico, habilidades técnico-prácticas y sentido pedagógico para implementar lo que la Reforma propone. Predominan todavía las actividades repetitivas, memorísticas, y los niveles de exigencia son bajos.

La investigación permitió comprobar que las prácticas educativas se encuentran todavía muy lejos de las deseadas, por lo cual es fundamental elevar el perfil profesional de los docentes y brindar mayor autonomía a las escuelas en la búsqueda y promoción de nuevas modalidades de desarrollo profesional permanente.

Lamentablemente, el estudio no incluyó análisis que permitan comparar las prácticas de las escuelas públicas con las de las privadas. No es posible, por lo tanto, argumentar que las diferencias de prácticas pedagógicas expliquen en parte las disparidades de rendimiento entre los alumnos de distintos sectores socioeconómicos. Pero sí es posible afirmar que en el sector público se promueve muy poco una educación que desarrolle las destrezas demandadas por los sectores sociales y productivos. Aunque hay avances, sobre todo en el plano normativo, las prácticas son todavía muy tradicionales. Las acciones emprendidas desde el 
Ministerio de Educación para mejorar la enseñanza tienen de hecho un impacto mucho menor que el esperado. Por ejemplo, las capacitaciones a los docentes parecen haber modificado poco sus prácticas y sus fundamentos pedagógicos: los libros y las bibliotecas que ahora existen son utilizadas fundamentalmente para que los niños transcriban del texto al cuaderno. El nuevo currículo se aplica, pero sin rescatar el sentido de las metodologías propuestas.

A las debilidades ya mencionadas de las escuelas públicas, se suman carencias que acrecientan mucho la distancia con las escuelas privadas. Por ejemplo, el acceso a computadoras para los alumnos/as del nivel básico y medio es todavía una quimera en el sector público, mientras es una realidad desde hace varios años en muchas escuelas privadas. El acceso a Internet en la escuela tampoco es una posibilidad real para los estudiantes y docentes del sector público. Laboratorios que permitan impulsar interés por las ciencias y el conocimiento científico son escasos incluso en las universidades y, por supuesto, aún más en la educación básica y media del sector oficial. El aprendizaje de un segundo idioma en la escuela pública tampoco es factible, mientras en muchos colegios privados sus estudiantes se gradúan hablando una segunda lengua y, en algunos casos, una tercera.

\section{$\mathrm{V}$}

\section{Los límites de la educación y la maximización de sus aportes}

En los años noventa El Salvador comenzó la reconstrucción del país. Una de las acciones principales emprendidas en el ámbito social ha sido la Reforma Educativa, que comenzó fortaleciendo los primeros años del sistema de enseñanza y ha ido abarcando progresivamente los niveles superiores. Uno de los principales logros de este proceso ha sido la concertación de la preocupación nacional por mejorar las condiciones educativas. Al cabo de algunos años lo hecho en virtud de la Reforma se ha traducido en resultados importantes. Así, la cobertura educativa ha crecido significativamente y de manera sostenida, con un sesgo positivo en favor de los sectores más pobres. Pero a pesar de los avances, persisten importantes limitaciones de acceso, sobre todo a la enseñanza parvularia, media y superior, especialmente para las personas con menos recursos. También se han aplicado medidas para mejorar la calidad, aunque su impacto ha sido menor que el deseado y, debido a los grandes problemas de la sociedad y del sistema educativo, todavía queda mucho camino por recorrer.

La educación se plantea como una de las principales estrategias para lograr la transformación productiva y un crecimiento económico suficiente y sostenido que permita que la población supere la pobreza y tenga mejores condiciones de vida. Sin embargo, la pobreza de las familias limita en buena medida las oportunidades educativas. La educación sólo podrá ayudar a que el país alcance mayores niveles de desarrollo en la medida que la población pueda recibir una buena educación, cualquiera sea su situación económica. La idea es generar condiciones que permitan que todos los sectores sociales tengan iguales oportunidades educativas, cosa que hoy no ocurre.

El país está todavía muy lejos de maximizar el aporte de la educación al desarrollo. Aunque el sistema educativo ampliara su cobertura al punto de que hubiera suficientes escuelas y profesores, y la educación que se recibiera en la escuela fuera de la más alta calidad, muchos de los niños/as no podrían asistir a la escuela porque sus familias son pobres. Pese a que la escuela es gratuita, un buen porcentaje de las familias del país se enfrentan con la necesidad de generar los ingresos indispensables para satisfacer sus necesidades básicas. En los sectores más pobres, los niños/as y jóvenes que no asisten a la escuela lo hacen no sólo por la falta de una oferta educativa adecuada, sino porque necesitan obtener ingresos para poder subsistir. Otros tantos, aunque logran llegar a la escuela, no cuentan con la misma red de respaldo de los niños/as con mejores condiciones económicas y, por lo tanto, su aprovechamiento y rendimiento son menores. En el hipotético caso de que el sistema educativo lograra que todos los niños y niñas, independientemente de su condición económica, asistieran a la escuela, todavía quedaría por superar la enorme inequidad en la calidad de 
la educación recibida por pobres y ricos, así como la disparidad de capacidades para aprovechar las oportunidades de aprendizaje.

El sistema educativo enfrenta el reto de mejorar su funcionamiento interno, es decir, ampliar su cobertura y mejorar su calidad, pertinencia y eficiencia, teniendo en cuenta criterios de equidad. Desde la perspectiva del desarrollo, esto contribuiría de un modo importante a que hubiese crecimiento y progreso. Sin embargo, los aportes de la educación al desarrollo tienen un límite que se traza donde la influencia de otras variables sociales comienza. Por lo tanto, para mejorar la condición de la población salvadoreña se necesita además un conjunto de otras medidas encaminadas a superar la pobreza y la inequidad. No se trata de resucitar disputas sociales violentas, sino de generar estos cambios dentro de la convivencia democrática.

La polarización que el país vivió en los años ochenta y el derrumbe de ciertas propuestas sociales extremas han hecho que, paradójicamente, en un país pobre como El Salvador, con grandes disparidades, se hayan postergado ciertos temas sociales de carácter estructural ante la necesidad de comenzar un legítimo proceso democrático, de llevar adelante la reconstrucción y de mantener una economía estable. Para que el modelo económico actual funcione de manera óptima, todos los integrantes de la sociedad deben disfrutar de igualdad de oportunidades; de lo contrario, el sistema sería altamente injusto y sostendría las desigualdades, aunque lentamente muchas familias superaran mínimamente el umbral de la pobreza. En las acciones del gobierno debe asignarse un lugar muy importante a la educación, pero en el contexto de un conjunto de medidas en los ámbitos social, económico, político, legal y cultural que formen parte de una política integrada de crecimiento y desarrollo. No sería sano para la sociedad salvadoreña sobreestimar, ni tampoco subestimar, la importancia de la educación en el desarrollo.

Ante la multidimensionalidad de la tarea que enfrenta esta sociedad, la educación busca transmitir el conocimiento y las destrezas de una generación a otra no sólo para reproducir la herencia de la generación anterior, sino para elevar el nivel de conocimiento y la condición de vida de las personas: debe contribuir así al cambio que, en el caso de El Salvador y de los países latinoamericanos, significa buscar modos de convivencia social más justos para todos. Este es el papel transformador de la educación, que debe estimular a las personas a reflexionar, efectuar ajustes y crear propuestas sociales, económicas, políticas, científicas y culturales. Esta manera de entender la educación, que puede parecer una mera consideración conceptual, en realidad dista mucho de ser sólo eso. Una educación comprometida con el desarrollo de la sociedad y de los sectores más pobres tiene aplicaciones concretas en los currículos y en las prácticas educativas a todos los niveles.

Al replantearse en El Salvador los fines de la educación, es necesario incluir entre ellos el de promover destrezas y hábitos de participación política en un contexto democrático y de libertad. La educación, no el hambre, debe ser el vivero de la conciencia social y política. También es importante tener en mente que la educación cumple un importante papel en la realización personal, lo que la hace valiosa en sí misma, aparte cualquier consideración relacionada con el mercado. Debe tenerse siempre en cuenta que la educación es un derecho humano.

A las necesarias consideraciones acerca del modo de entender la educación y su aporte real al desarrollo del país, cabe añadir que el sistema educativo salvadoreño dista mucho de maximizar su contribución a la sociedad. Para que el sistema pueda cumplir con sus metas son necesarias varias medidas como las que se describen a continuación.

En la búsqueda de soluciones creativas y bien fundamentadas a los problemas de la educación en El Salvador, es importante elevar la capacidad técnica del Ministerio de Educación. Sería útil apoyar significativamente el desarrollo profesional de sus técnicos, de los profesores e investigadores de las universidades, así como de otros profesionales de organizaciones no gubernamentales que trabajen en el plano educativo. Es aconsejable revisar las estructuras organizativas, burocráticas y de incentivos del Ministerio y otras instituciones, con el fin de estimular el perfeccionamiento profesional y la investigación.

Relacionado con lo anterior, es preciso generar un debate nacional entre profesionales en torno a los datos que el sistema origina, a las ideas y a las propuestas. Para eso, la información clave que maneja el Ministerio de Educación -por ejemplo, los resultados de las pruebas de rendimiento de la educación básica y de la Prueba de Aprendizaje y Aptitudes para Egresados de Educación Media- debe ser del dominio público y producto de un proceso transparente. Sin duda hay un riesgo de que los resultados se utilicen políticamente en favor o en contra del gobierno, pero también es cierto que no podrá haber reflexión para buscar soluciones si esos resultados no se informan. Por otra parte, los ciudadanos e instituciones sociales tienen derecho a conocer el funcionamiento de los siste- 
mas públicos. Es necesario, por lo tanto, fortalecer el apoyo a la investigación social y a la evaluación del rendimiento del sistema educativo.

Asimismo, se debe continuar ampliando la cobertura, sobre todo hacia los sectores rurales y las regiones más pobres. Aunque se ha hecho mucho en el nivel básico, se precisan ahora iniciativas de expansión dirigidas al nivel preescolar y a la educación media. También es esencial elaborar un conjunto de medidas que garanticen la continuidad en los niveles de educación postsecundaria para los estudiantes más pobres. Además, en todos los niveles habrá que desarrollar servicios educativos de calidad para los estudiantes de menos recursos. El sistema de educación formal tradicional responde a supuestos sociales distintos a los que enfrenta gran parte de la población: asume, por ejemplo, que todos los alumnos tienen el respaldo necesario para que puedan dedicar su tiempo a asistir a la escuela. Sin embargo, como esa situación ideal no se da en un sector considerable de la población y, en el mejor de los casos, probablemente no se dará por algunas décadas, es aconsejable que los educadores busquen nuevas modalidades para llevar la enseñanza a los sectores más pobres.

Por otro lado, buena parte de la población laboralmente activa deja de estar vinculada con el sistema educativo formal. A modo de ejemplo, el $14 \%$ de la población salvadoreña tiene entre 20 y 30 años, fue educado en el sistema educativo anterior a la reforma, y solamente el $11 \%$ de este sector poblacional está estudiando (El Salvador, Dirección General de Estadísticas y Censos, 1998). Por lo tanto, es indispensable coordinar esfuerzos para brindar a la población mayor de 20 años oportunidades de capacitación relacionadas con la productividad.

En lo que se refiere a mejorar la calidad de la educación, una de las medidas iniciales deberá ser la de elevar el nivel de profesionalización de los docentes. Numerosas actividades de capacitación efectuadas a partir de 1990 han hecho aportes, pero han exhibido aspectos cuestionables y han tenido escaso impacto en las prácticas educativas (FEPADE, 1998). Urge buscar para los 28 mil maestros en servicio nuevas modalidades descentralizadas de desarrollo profesional económicamente sostenibles y más apegadas al modelo pedagógico que se desea que los profesores practiquen con sus alumnos. Con el tiempo, podrían incorporarse a ellas la tecnología y la educación a distancia.

La formación de docentes fue reestructurada recientemente, y el cambio ha sido bien recibido por la mayoría de los sectores. Por cierto que estos cambios deben ser evaluados continuamente para detectar dificultades. Por otra parte, las facultades de educación de las universidades deben ser apoyadas con más recursos y con oportunidades de desarrollo profesional para los catedráticos universitarios, lo que a su vez permitiría elevar el perfil de los profesionales que las universidades forman y la calidad de su trabajo.

Los bajos resultados en las evaluaciones nacionales del aprendizaje de lenguaje y matemáticas indican que es preciso tomar medidas específicas, de distinta naturaleza, para ir superando progresivamente estas deficiencias. La investigación y experimentación pedagógicas sobre estos temas son determinantes para diseñar políticas apropiadas. ${ }^{6}$

El fenómeno de la repitencia en El Salvador también debe investigarse más para conocer sus principales causas en los sectores más pobres. Los recursos del sistema educativo y las oportunidades de aprendizaje de los alumnos se verían muy beneficiados si se aplicaran políticas orientadas específicamente a atacar este problema.

El sistema educativo ha iniciado con cierto éxito el proceso de descentralización; ha transferido competencias administrativas a las regiones y, en los últimos años, a las escuelas mismas. Sin embargo, la delegación de autoridad actual no basta para que cada escuela geste su perfil institucional, ni para definir, adecuar o fortalecer los procesos pedagógicos (Carrasco, Guevara, Hernández y Rodríguez, por publicarse). En otros países la descentralización de la gestión escolar y pedagógica al nivel de las escuelas ha creado dinámicas de trabajo en equipo y la posibilidad de generar actividades más pertinentes y creativas (Chile, MINEDUC, 1998). Una medida atinada que el Ministerio de Educación podría aplicar es la de proporcionar mecanismos, financiamiento y asistencia técnica para proyectos escolares gestados en equipo, que involucren a la comunidad y que presenten propuestas valiosas e innovadoras. Medidas de este tipo pueden tener efectos importantes, ya que junto con generar una cultura de trabajo distinta en los centros educativos, abren la posibilidad de que el sistema aprenda desde abajo, desde las escuelas. La descentralización escolar en El Salvador debe propiciar, en el mediano plazo, mayor autonomía en los centros educativos (Carrasco, 1998). Por supuesto que el proceso de descentralización, para ser efectivo, debe ir acompañado de apoyo técnico

\footnotetext{
${ }^{6}$ En 1999, el FEPADE abrió por segundo año consecutivo un fondo de investigaciones para el aprendizaje de la lectoescritura y las matemáticas.
} 
oportuno y de recursos financieros que hagan viables las iniciativas generadas en los centros.

Una mirada a la política de descentralización hacia las escuelas revela que la posición del director de la escuela es clave para fomentar la toma de decisiones participativa, el trabajo cooperativo y la gestión autó- noma. Es necesario entonces promover instancias de desarrollo profesional específicas para los directores de los centros escolares, en áreas tales como liderazgo, participación, elaboración de proyectos, evaluación y relación escuela-comunidad (Carrasco, 1998; Carrasco, Guevara, Hernández y Rodríguez, por publicarse).

\section{Bibliografía}

BID (Banco Interamericano de Desarrollo) (1998): Basic SocioEconomic Data, El Salvador, ESDB Query Facility

Carrasco, A. (1998): Crónica analítica. Comentarios en torno a la presentación del estudio 'El Salvador Reforma Educativa'. Análisis del proceso de descentralización. San Salvador, El Salvador.

Carrasco, A. y A. Fernández (por publicarse): La reforma educativa en El Salvador. Informe de El Salvador para el Programa de Promoción de la Reforma Educativa en América Latina y el Caribe (PREAL), San Salvador, El Salvador, Publicaciones FEDAPE, Colección Investigaciones.

Carrasco, A., M. Guevara, C. Hernández, y M. Rodríguez (por publicarse): Los consejos directivos escolares. Análisis de una estrategia de administración escolar local participativa. San Salvador, El Salvador, Publicaciones FEPADE, Colección Investigaciones.

CEPAL (Comisión Económica para América Latina y el Caribe) (1997a): Panorama social de América Latina, 1996, LC/G.1946-P, Santiago de Chile.

(1997b): La brecha de la equidad. América Latina, el Caribe y la cumbre social, LC/G.1954 (CONF.86/3, Santiago de Chile.

(1998): Panorama social de América Latina 1997. LC/G.1982-P, Santiago de Chile.

Chile, MINEDUC (Ministerio de Educación Pública) (1998): Manual para la educación básica. Proyectos de Mejoramiento Educativo, Santiago de Chile.

El Salvador, Dirección General de Estadísticas y Censos (1997): Encuesta de hogares de propósitos múltiples, EHPM 1996, San Salvador, El Salvador.

(1998): Encuesta de hogares de propósitos múltiples, EHPM 1997, San Salvador, El Salvador.

El Salvador, Ministerio de Educación (1995): Documento III. Lineamientos Generales del Plan Decenal 1995-2005, San Salvador, El Salvador.
(1996): Informe de evaluación del rendimiento en $3^{\circ}$ y $5^{\circ}$ grados de Educación Básica en lenguaje, matemáticas, estudios sociales y ciencia, salud y medio ambiente, basado en la aplicación nacional de pruebas de octubre de 1995, San Salvador, El Salvador.

FEPADE (Fundación Empresarial para el Desarrollo Educativo) (1997): Los jóvenes en situación de exclusión social. Caracterización de la niñez y adolescencia de 7 a 18 años de El Salvador, San Salvador, El Salvador

(1998): Memorándum analítico. En la búsqueda de nuevos modelos de desarrollo profesional permanente. San Salvador, El Salvador.

FEPADE/El Salvador, Ministerio de Educación (1998). Memorándum analítico. Factores asociados al rendimiento. Datos de las Pruebas de Logros de Aprendizaje de Lenguaje y Matemáticas. Base de datos de 1996. San Salvador, El Salvador.

IUDOP/UCA (Instituto de Opinión Pública/Universidad Centroamericana José Simeón Cañas (1997): Encuesta sobre el sistema educativo y la salud, San Salvador, El Salvador.

PIIE/UNICEF (Programa Interdisciplinario de Investigaciones en Educación)/(Fondo de las Naciones Unidas para la Infancia) (1995): Equidad y educación básica en Chile, Análisis de los resultados del SIMCE en $4^{\circ}$ año básico 1992, Santiago de Chile.

PNUD (Programa de las Naciones Unidas para el Desarrollo) (1997): Informe sobre desarrollo humano, 1997, Nueva York.

(1998): Desarrollo humano en Chile 1998: las paradojas de la modernización, Santiago de Chile.

PROFINE (Programa de Formación de Investigadores Educativos) (1997): ¿Tú aprendes? ¿Yo enseño? Discurso y realidad en las escuelas salvadoreñas, San Salvador, El Salvador, publicaciones FEPADE.

Reimers, F., coord. (1995): La educación de cara al siglo XXI. Desafíos y oportunidades, San Salvador, El Salvador, Universidad Centroamericana José Simeón Cañas/UCA Editores. 